\title{
NATURE AND MEASUREMENT OF WHITENESS
}

$\mathrm{T}$ HE mechanism by which a 'white' substance reflects light is fairly simple and is fundamentally the same for crystalline and vitreous powders such as snow and borax, for suspensions such as milk, paint and opal glass, and for fabrics and paper. In all these cases, a multiplicity of transparent particles or fibres is suspended in a transparent medium of different refractive index, and a beam of light incident on the substance is specularly reflected and refracted at a large number of random interfaces, without any transformation and with very little absorption, resulting in a diffused reflexion. A coloured substance has the same mechanism, except for interference colours, but the minute particles also exert selective absorption on the light passing through them. If the difference of the refractive indexes between the particles and the surrounding medium is reduced, a white substance will have a reduced reflexion factor owing to reduction of the interfacial reflexion, and for the same reason a coloured substance will have an increased coloration, as may be simply shown by wetting a white or a coloured fabric. No reflecting substance has colour of its own, but only introduces colour by virtue of the selective absorption which it exerts on light falling upon it, and a white substance is uniquely characterized by its absence of selective absorption of light.

A 'white' light has no such unique character, and the range of lights which are called white can only be defined subjectively from the white-hot netal in a furnace at a temperature of about $1,700^{\circ} \mathrm{K}$. to north sky daylight the colour temperature of which may be $10,000^{\circ} \mathrm{K}$. It is, however, essential that standard white illuminants should be employed for measurement of white and of coloured substances, and ten years experience of rlluminants $A, B$ and $C$ specified by the Commission Internationale d'Éclairage in 1931 has proved them adequate for most purposes.

The Colour Group of the Physical Society held a discussion on whiteness on September 24, and three papers were read on different aspects of the problem of measuring near-whites. The introductory paper by J. G. Holmes, of Messrs. Chance Brothers and Co., Ltd., gave the theoretical background and indicated some of the visual and colorimetric problems peculiar to near-whites. It was pointed out that a white substance can be picked out quite critically either in daylight or in artificial light, whereas a particular yellow or blue calls for an effort of memory and for uniformity of lighting, and this sensitivity to small departures from whiteness is stimulated by the frequency with which white and near-white substances are employed in everyday life. The exact measurement of their colour is more exacting than that of coloured substances, and instrumental methods may be hard taxed to detect and to measure differences which are quite obvious to the unaided eye. The amount of departure from whiteness is usually expressed as the difference between the colour of the light reflected from a substance and the colour of the light incident upon it; a truly white substance making no change in the colour of the light.

A brief review was given of the instruments available and of their usefulness in industry. The usual type of trichromatic colorimeter is not satisfactory, as the method of building up a white light by adding together three strongly coloured primary lights pro- duces a very uneven spectral energy distribution and, when this is compared with the light reflected from the specimen, the difference in energy distribution in the two halves of the colorimeter field renders the match liable to appreciable variation from one observer to another. This disadvantage may be reduced by employing a subtractive colorimeter, in which the selective absorption of the specimen is approximately reproduced by that of the subtractive filters, but all visual colorimeters have a small field size, leading to poor sensitivity. Spectrophotometric measurements are free from these disadvantages and good results can be obtained by visual or photoelectric 'abridged spectrophotometers', which measure reflexion factor in eight or more comparatively narrow spectral bands selected by filters, and by complete spectrophotometers which measure rereflexion factor in monochromatic light at a series of wave-lengths.

Dr. V. G. W. Harrison, of the Printing and Allied Trades Research Association, dealt with colour tolerances and methods of control in the paper industry. The natural colour of paper is yellowish, its hue being about $5800 \mathrm{~A}$. and its saturation about 12 per cent with C.I.E. Illuminant $B$; the paper trade tends to call this 'white', and to call a true neutral pulp 'blue-white'. There is a marked preference for the latter colour which is not due to any tradition in the trade, and Dr. Harrison described experiments in which twenty observers were asked to compare the brightness of eight different papers. Analysis of their results showed that the physical reflexion factor of a paper is no measure of its brightness by subjective estimation, and that a blue. white paper actually appears brighter than a natural white paper the reflexion factor of which is about 6 per cent higher than the blue-white.

It seems that the eye is more sensitive to changes in saturation, or depth of colour, than in reflexion factor, and a paper is judged brighter if it has less selective absorption, which explains the common practice of a beaterman in a paper mill, who adds a dash of blue or black to his pulp to 'brighten' the paper. The accepted commercial tolerances are very close when papers have to be bound togother in a book, and Dr. Harrison referred to a single run of paper from one mill which had to be graded visually into six 'colours' in order to ensure consistency. Colorimeter measurements failed to show any significant difference between the six grades, and in order to get sufficiently accurate data it was necessary to send the specimens to the United States for measurement on a recording photo-electric spectrophotometer. Subsequent tests on a photo-electric 'abridged spectrophotometer' gave very similar results, although these were not suitable for direct calculation of the trichromatic coefficients, and the tolerance for good commercial quality appeared to be closer than \pm 0.0005 in the C.I.E. trichromatic coefficients and \pm 0.5 per cent in the reflexion factor.

A manufacturer has to work to a standard pattern, and it is very difficult to check gradual change due to dirt and ageing without accurate measurement. An appeal was made for the provision of a rapid and accurate spectrophotometer available for frequent and inexpensive industrial testing of patterns, 
preferably under the auspices of a testing centre or research association.

C. G. Heys-Hallett, of the Morgan Crucible Co., Ltd., discussed the apparent whiteness of cinema screens. Coloration of the screen itself and the colour of the light from the arc are found to vary widely in many cinemas and to lead to serious distortion in the excellent colour films of the present day. This distortion can be overcome by standardization, but it is first necessary to measure the apparent colour and brightness of the combination of arc, optical projection system and screen in order to demonstrate to cinema proprietors the necessity for uniformity. Such measurements need not be on a standard system and do not need the high accuracy required in the paper industry, and Mr. Heys-Hallett described a photo-electric brightness meter which indicates screen brightness with no film in the projector and the relative brightness with each of three colour filters interposed in the beam.

The variation from cinema to cinema is large, being more than $50: 1$ in screen brightness and about 30 per cent in colorimetric saturation, with the result that many films are shown at a disadvantage. The variation in brightness is attributable to the use of the most powerful arc lamps in some of the smaller modern cinemas and unsuitable projectors in some of the older cinemas, rather than to the reflecting properties of the screen; whereas the variation in colour is due to the use of low-intensity and highintensity arcs, the variety of mirror and lens collecting systems and the rapid ageing of the screen. It is interesting that the use of a blue filter in the projector to correct a yellowish colour is a definite disadvantage, contrary to experience in the paper industry, as the loss of screen brightness causes more subjective colour distortion than the partial correction given by the blue filter.

Demonstrations were given by lantern slide, and it was suggested that if the projected colour and brightness could be standardized at any practical value, colour films could be made to suit. Such a standardization could fairly easily be applied also in the United States and on the Continent, but it would restrict the development of huge super-cinemas until more powerful light sources are available.

\section{THE 'G.E.C. HEAVY ALLOY'}

$I^{\mathrm{N}}$ an article on the new 'G.E.C. Heavy Alloy' published in the G.E.C. Journal of August, G. H. S. Price and S. V. Williams, of the G.E.C. Research Laboratories, in conjunction with C. J. O. Garrard of the Witton Engineering Works, describe the preparation, properties and industrial uses of a tungsten alloy which is fifty per cent heavier than lead and has the tensile strength of a good-quality steel.

Of the common metals, lead is the only one which is substantially heavier than the general engineering materials. Lead, with its excellent corrosion-resisting properties, is a very useful metal, but it is weak mechanically, and for this reason its use is limited to those applications where the stresses are low. The precious metals such as gold or platinum are heavy metals, being two to three times as heavy as iron or copper, but their high price prohibits their use for general engineering purposes. Tungsten, however, has a density equal to that of gold and is also relatively cheap. A considerable tonnage of tungsten is used annually as an alloying element in the production of special steels, and for this purpose the metal is added in the form of powder or as a ferro-tungsten alloy. Tungsten is characterized by a very high melting point $\left(3,400^{\circ} \mathrm{C}\right.$.) and this precludes the production of the pure metal by ordinary metallurgical processes. Pure tungsten in the form of ductile wire or sheet such as is used in the manufacture of electric lamps or radio valves, is produced by a powder metallurgical process. The successful development of the process about thirty years ago was one of the first and most important examples of this method of producing metals and alloys, a method which has been extended in many directions, particularly during the last few years.

So far as tungsten is concerned, a bar of pressed powder is sintered to a high temperature and afterwards worked by swaging, drawing or rolling to produce the metal in wire or sheet form. The sintering temperature required is in the region of $3,000^{\circ} \mathrm{C}$. and this is obtained by passing an electric current (about 2,000 A. per 1/16 sq. in.) through the bar. The method of sintering, however, limits the size of the bar that can be conveniently handled, and having regard to the fact that it is only after a considerable amount of work has been done on the sintered bar that the metal approaches its theoretical density, it is evident that fully dense tungsten cannot be produced in massive form.

In the first instance, attempts were made to produce an alloy of tungsten and lead. Tungsten powder was mixed and pressed with a sufficient quantity of lead powder to fill the interstices between the tungsten particles, when the mixture was heated. It was found, however, that tungsten and lead do not wet each other, and homogeneous masses could not be produced. It was known that nickel and tungsten alloy very readily, and experiments were made using mixtures of tungsten powder with 5-10 per cent nickel. On pressing and sintering to a moderate temperature, alloys were obtained with densities of the order of $16 \cdot 5-17 \mathrm{gm} . / \mathrm{c.c} .$, which is more than twice that of steel $(7 \cdot 8)$ and about 50 per cent greater than lead. It was then found that the addition of a certain amount of copper assisted production, and although the quantities of nickel and of copper can be varied, the material which is known as 'Heavy Alloy' usually contains 90 per cent tungsten, $7 \cdot 5$ per cent nickel and $2 \cdot 5$ per cent copper. In addition to its high density, 'Heavy Alloy' has a tensile strength comparable with that of a good quality steel.

It is an interesting fact that although the light alloys of aluminium and magnesium are playing such a leading part in modern aircraft construction, it is in this field that ever-increasing use is being made of 'Heavy Alloy'. It is well known that tungsten is practically unmachinable, but 'Heavy Alloy', although it contains about 90 per cent tungsten, has excellent machining properties. The development of 'Heavy Alloy' with a tensile strength of 40 tons/sq. in. and a density more than twice that of steel opens up new possibilities for the designer. 